\title{
Engineering the Lymphatic System
}

\author{
Matthew E. Nipper and J. Brandon Dixon \\ Woodruff School of Mechanical Engineering, Georgia Institute of Technology, Tel.: \\ +404-385-3915, Fax: +404-385-1397 \\ J. Brandon Dixon: dixon@gatech.edu
}

\begin{abstract}
The recent advances in our understanding of lymphatic physiology and the role of the lymphatics in actively regulating fluid balance, lipid transport, and immune cell trafficking has been furthered in part through innovations in imaging, tissue engineering, quantitative biology, biomechanics, and computational modeling. Interdisciplinary and bioengineering approaches will continue to be crucial to the progression of the field, given that lymphatic biology and function are intimately woven with the local microenvironment and mechanical loads experienced by the vessel. This is particularly the case in lymphatic diseases such as lymphedema where the microenvironment can be drastically altered by tissue fibrosis and adipocyte accumulation. In this review we will highlight contributions engineering and mechanics have made to lymphatic physiology and will discuss areas that will be important for future research.
\end{abstract}

\section{Keywords}

lymphatic; biomechanics; lymphedema; imaging; shear stress; tissue engineering

\section{Introduction}

The lymphatic vasculature was reported as early as 1564 and has long been appreciated as essential to maintaining fluid balance. However, the last decade has produced a renaissance of sorts in our understanding of the lymphatics' active role in immune cell trafficking [1], lipid transport [2], and pathological processes like cancer and tissue inflammation [3,4]. Lymphatic vessels begin in the interstitial spaces throughout the body where they continuously drain fluid and proteins into the initial lymphatics. These initial lymphatics merge together downstream into larger collecting lymphatics that actively contract to pump fluid through the lymph nodes and the lymphatic network to enter the blood near the heart. Recent estimates suggest that lymphatics in humans return as much as 8 liters of fluid back to the blood per day [5]. While we certainly cannot discount the role that modern molecular biology has played in advancing our understanding of the lymphatics, we believe that a multi-disciplinary, bioengineering approach has made and will continue to make a substantial impact on the field.

Advances in imaging and tissue engineering are leading to the discovery of new physiologic functions in lymphatics. Studies in lymphatic biomechanics are demonstrating that the lymphatics have a finely tuned response to mechanical load that can rapidly adapt to local changes in pressure and fluid flow. Mathematical models of individual lymphatics and lymphatic networks are now being developed that capture some of these complexities. Advancing these methods will be particularly important to diseases with lymphatic 
involvement, such as lymphedema and cancer metastasis, where changes in the mechanical microenvironment of the lymphatics are likely to have profound effects on lymphatic function. In this review we will summarize and highlight the major contributions bioengineering has made to our understanding of lymphatic physiology and biology: from novel experimental and imaging approaches to advances in lymphatic biomechanics and mathematical modeling. For the reader unfamiliar with the basics of lymphatic physiology we refer them to the following review by Swartz [6].

\section{Engineering tools for quantifying lymphatic function}

The discovery of lymphatic-specific growth factors [7] and antibodies for labeling lymphatic proteins [8] has sparked a recent resurgence in lymphatic research that has been furthered in part by the development of new techniques and engineered tools for quantifying lymphatic physiology and function. Traditional methods for measuring vascular hemodynamics have not been as effective in studying lymphatic flow due to the small size of the vessels, lack of optical contrast, and lower density of scatterers within the flow field. Most techniques rely on the inherent nature of lymphatics to drain large proteins from the interstitial spaces. By intradermally injecting various labeled tracers of specific sizes to provide contrast, researchers can visualize lymphatic architecture in vivo and estimate lymph flow rates through clearance of the tracer or appearance time in the draining lymph node [9]. When fluorescence imaging is combined with fluorescence recovery after photobleaching (FRAP) [10] or mathematical modeling [11], researchers have been able to measure actual velocities in the initial lymphatics draining the skin. By continuously injecting a very low dose of a fluorescent tracer at various pressure gradients, and allowing the uptake of the tracer to reach steady state, the contributions of both lymphatic conductance and tissue hydraulic conductivity to the transport of the tracer could be quantified [12]. This approach has proven to be valuable for measuring changes in both lymphatic function and the interstitium in various mouse disease models $[13,14]$.

While fluorescent tracers work quite well for measuring the constant draining velocity of the initial lymphatics, this approach is not useful for measuring the rapid fluctuations in lymph flow that occur in the highly contractile collecting lymphatics. Velocities of lymph flow throughout the entire contraction cycle have been measured by applying a high speed imaging system with an intravital microscopy preparation of the rat mesentery [15]. Subsequent automation of the image analysis of these videos [16], is allowing researchers to use this approach as a powerful tool for studying the functional lymphatic response in a variety of physiologic scenarios. Another promising imaging tool for imaging lymphatic function both in animals $[9,17,18]$ and humans [19-21], is the use of near-infrared (NIR) fluorescence imaging. By injecting an NIR tracer intradermally, researchers have shown the ability to non-invasively visualize lymph transport. This technique should prove quite useful clinically for evaluating the immediate efficacy of new lymphedema treatments, as a clinically approved NIR dye (ICG) already exists and has been used in the clinic for opthalmic angiographies for over 30 years. However, concerns exist in regards to the deleterious effects of ICG on lymphatic pump function, and whether or not such an approach is warranted as a diagnostic tool, particularly in patients at risk for lymphedema [22].

In addition to imaging, there have been several other technical advances made in the field that are furthering our understanding of the lymphatics. Tissue culture, either by vessel isolation, or by building from the "bottom up" of environments that contain the essential biological components (e.g. cell types \& extracellular matrix), provides the ability to manipulate the biology and the mechanical properties. Such approaches allows one to study the underlying mechanisms regulating function, while keeping the cell in an environment similar to its in vivo context. Whole vessel approaches through the cannulation, and 
mechanical and biological manipulation of isolated lymphatics have been practiced for over 30 years. Gashev and colleagues have added to this toolbox by developing a gene transfection protocol for isolated rat lymphatic vessels [23]. This technique will be particularly useful for understanding the transcriptional control of lymphatic smooth muscle and lymphatic endothelial cells (LECs) in regulating pump function. This is important given that the rapid tonic contractions of lymphatic vessels demonstrated in humans appear to be lacking in mice, making transgenic mice less than ideal for studying smooth muscle control of lymphatic transport [24]. Lymphatic vessel cannulation in humans has also shed light on the role of lymphatics in lipid transport from the periphery $[25,26]$. These techniques, coupled with advances in pro-teomics, will be essential for pushing forward our understanding of lymphatic transport as it relates to health and disease since it is highly likely that lymph is more than just an ultrafiltrate of blood plasma [27].

Advances in engineering will continue to increase the complexity and physiological relevance of other lymphatic "organ culture" approaches. For example, the Uhlig research group successfully cultured a loop of the small bowel for up to 4 hours while sampling and permitting analysis of the vascular, luminal, interstitial, and lymphatic compartments simultaneously [28]. Cannulation and perfusion of individual vessels does not lend itself well to the large sample sizes often needed for certain biological studies, and it can take months to years of training to master the microsurgery skill-set needed to manipulate such small, fragile vessels. Thus, a bottom-up tissue engineering approach that contains the essential cellular components needed to recapitulate in vivo function would be advantageous. This approach was taken to develop a model for studying lipid transport from the intestine into lymphatics [29], an essential function of the lymphatic system as nearly all dietary lipid is transported into the circulation by the lymphatic system [30]. Tissue engineering approaches have also provided in vitro evidence for the molecular and biophysical cues that are involved in lymphangiogenesis [31-33].

\section{Lymphatic Biomechanics}

The lymphatic vasculature encompasses a majority of tissues throughout the body, promoting one-way transport of fluid from the interstitium back into the venous circulation. To achieve this, the vessels must move fluid up a pressure gradient, produced from interstitial fluid pressure (which is often negative), gravity induced hydrostatic pressures, and central venous pressure. This feat is accomplished through the very specialized morphology of the initial draining lymphatics, the intrinsic pumping capabilities of the down-stream collecting lymphatics, and the presence of valves throughout the vessels preventing back-flow. (For a thorough review on lymphatic physiology and anatomy see [34]). Briefly, the most basic element is the initial lymphatic, having an open-ended microvessel structure with coherent specialized junctions that both allow for easy fluid entry upon tissue expansion and prevent back-flow [35,36]. The initial lymphatics terminate into the collecting lymphatics which are comprised of individual sub-units separated by valves termed lymphangions. The inherent pumping action of collecting lymphatic vessels is facilitated through the lymphangions, which are lined with specialized smooth muscle cells capable of regulating both vessel tone and promoting rapid contractions of the vessel [37, 38]. Valve leaflets separate each lymphangion resulting in net unidirectional flow during the pumping cycle and minimizing backflow upon subsequent vessel expansion (Figure 1).

The contractile profile of the lymphatic is not predetermined and has been shown to be quite sensitive to changes in the mechanical loads subjected during lymph transport. Given that lymph formation can vary quite substantially during routine physiologic functions [39], it seems likely that lymphatics would be mechanically sensitive to their local environment, altering their behavior appropriately to optimize lymph transport [40]. This capacity of a 
lymphatic to serve as a pump or a conduit is a result of a highly sensitive response to changes in the mechanical forces acting upon the vessels. Observations of lymphatic vessels in vitro, in situ, and in vivo have revealed a wealth of information about this mechanical sensitivity.

\section{Tensile forces in lymphatic vessels}

Early experiments with cannulated collecting lymphatic vessels from cow, rat, and guinea pig models show that modulating intraluminal pressure results in dynamic shifts in pump frequency and stroke volume $[41,42]$. The contractility during the pump cycle, which consists of 2 systolic phases coupled with 4 diastolic phases [43], is quite impressive allowing for contractions of up to $80 \%$ of the resting diameter [37,38,44]. Additionally, increasing lymph volume via plasma dilution was shown to increase contraction frequency and lymphatic diastolic vessel diameter in vivo [43]. Not only does the magnitude of stretch affect the lymphatic myogenic response, but this response has been shown to be highly sensitive to the rate of change of the load applied to the vessel [45]. Such rate-sensitive adaptations were different than those seen in the portal vein, suggesting that the lymphatics have adapted to compensate for the rapidly varying changes in load that can occur in these vessels in vivo.

Ultimately lymphatic flow is promoted by multiple lymphangions working in tandem to propel fluid through the lymphatic system. Thus, Zawieja and colleagues sought to measure the propagation of these contractions in vivo [46]. It was shown that inter-lymphangion contractions are both propagated and coordinated and that the frequency of contractions is equally distributed along the length of the vessel with this coordination facilitated by gap junction communications. Previous experiments validated this hypothesis by loading individual lymphatic compartments with heptanol (a known gap junction inhibitor) to impede contractile wave propagation through the segment [47]. A transfer efficiency of 55\% was found with the employed blockers compared to 80-90\% with control treatment. Additionally, external neuronal mediated depolarization events have not been found to play a significant role [47]. More recently, a high speed imaging system was reported that allowed automation of the tracking of contractile waves in lymphatics for determining the location of the pacemaker cells within the vessel responsible for coordinating lymphatic pumping [48]. The ability to identify pacemaking cells through such an approach will be beneficial for elucidating the molecular mechanisms that regulate this pacemaking and the consequences of dysfunctional pacemaking cells to lymph propulsion and lymphedema resolution.

The intrinsic pumping function of collecting lymphatic vessels is facilitated by multiple signaling cascades. Collecting lymphatics are often compared to cardiac muscle because of the autocontraction behevior that drives fluid transport. During contraction in cardiac muscle, an intracellular $\mathrm{Ca}^{2+}$ flux occurs to sustain depolarization of the muscle cell. Similarly, an intracellular calcium flux in a lymphatic vessel is observed during stretch induced contractions [49]. Multiple adrenergic signaling events are present in lymphatic contractions in which $\mathrm{Ca}^{2+}$ flux is triggered. Intracellular $\mathrm{Ca}^{2+}$ flux has been linked with a flux in chlorine anions which may act as a pacemaker mechanism [50,51]. However, the extent that these pathways are involved in mechanotransduction remains unexplored. $\mathrm{Ca}^{2+}$ influx is observed prior to reaching the maximum tension during the contraction event. As the intraluminal pressure is increased via stretch conditions, both the frequency and amplitude of spontaneous contractions increase. The close relationship between tensile deformation and $\mathrm{Ca}^{2+}$ flux was investigated using caffeine and cyclopiazonic acid, which are known intracellular $\mathrm{Ca}^{2+}$ modulators. Both caffeine and cyclopiazonic acid inhibit contractility under increasing transmural pressures [52]. The sensitivity of lymphatic vessels to $\mathrm{Ca}^{2+}$ flux has been experimentally compared to that of arterioles and veins [53]. 
Lymphatic vessels exhibit a lowered sensitivity to $\mathrm{Ca}^{2+}$ compared to arterioles, which may be reflected in the sole expression of myosin heavy chain $\mathrm{B}$, whereas arterioles commonly express both the $\mathrm{A}$ and $\mathrm{B}$ isoforms.

It is evident that tensile force propagation through lymphatic vessels is directly influenced by multiple mechanotransductive pathways. Elucidating these mechanisms will aid not only in characterizing lymphatic transport, but could provide insight on how they are altered in lymphatic disease, where vessels can experience prolonged abnormal mechanical loading [54]. Stretch is not the only force experienced by the cells of the lymphatic vessel and recent evidence suggests that shear stress is also an important mechanical cue for regulating lymphatic function.

\section{Wall shear stress}

The influence of wall shear stress (WSS) and its effects on the lymphatic vasculature is an emerging field of study. Similarities between the lymphatic system and blood vasculature suggest some overlap in response to shear stress. Modulating shear stress through flow rate has been found to induce nitric oxide (NO) release in lymphatic vessels $[55,40,23]$ in a mechanism similar to that of arterioles [56,57]. One question that arose from these isolated vessel studies was whether the flows and resulting shear stresses imposed in the experiments were within the range of those that occur in vivo. Subsequent in vivo experiments in the rat mesentery measured flow velocities throughout the entire contraction cycle for the first time and reported an in vivo estimation of WSS experienced by these vessels, demonstrating that the shear-induced response of isolated vessels was well within ranges of WSS that occur in vivo [37].

Recently, the biochemical pathways of this response have been investigated and revealed that NO release is driven by upregulation of endothelial nitric oxide synthase (eNOS) on LECs and is ATP dependent [58]. In particular, ATP is thought to target the puriginic P2X/ $2 \mathrm{Y}$ receptors which are ligand activated $\mathrm{Ca}^{2+}$ gates found in the plasma membrane. In this in vitro study on human LECs, the $\mathrm{P} 2 \mathrm{X} / 2 \mathrm{Y}$ receptors were activated upon a minimum threshold of $0.5 \mathrm{dyne} / \mathrm{cm}^{2}$. This shear stress dependent relationship observed in vitro for LEC's can be diminished by binding exogenous ATP with sumarin or blocking the P2X/2Y receptor. Upon receptor activation, an outward flux of $\mathrm{Ca}^{2+}$ is triggered leading to the activation of eNOS. A significant increase in eNOS was observed within 1 hour of flow initiation. Interestingly, the threshold for significant NO release in this study was 2.0 dyne/ $\mathrm{cm}^{2}$ whereas in isolated rat lymphatics it was shown that the vessels exhibited an NO dependent vasodilation at a WSS as low as $0.2 \mathrm{dyne} / \mathrm{cm}^{2}$ [40,37]. In either case, these NO responses to WSS are much lower than those reported in arterioles, which would be expected given that shear stresses experienced by LECs are typically much lower than those reported in the blood vasculature [37,59]. Others have also shown a reduction of the strength of lymphatic contraction in response to flow [60]. While that study also noted an increase in contraction frequency [60], several subsequent studies suggest that frequency decreases in response to elevated flow $[55,40,23]$. Such a response would also agree with computational approaches that have demonstrated that vessel contraction would increase vascular resistance in situations where an imposed flow gradient is already present $[61,62]$. Thus a shear-induced response that minimizes both the frequency and amplitude of contraction would be physiologically advantageous by reducing total lymphatic vascular resistance to flow.

The baseline biomechanic response (to both WSS and stretch) of lymphatics has been further compared to that of arteries and veins [63]. Lymphatics exhibit bimodal force development during the pumping phase compared to a monotonic force development for arteries. Lymphatics also have a peak active tension that is one-tenth the magnitude seen in a 
similar sized artery. The intrinsic differences in biomechanic action are a consequence of the difference in composition of lymphatic smooth muscle compared to arteries as lymphatics express higher levels of actin and myosin heavy chains [38]. It seems likely that lymphatic vessels would exhibit a plasticity similar to that of arteries and veins, which are known to initiate growth and remodeling in response to alterations in their mechanical environment [64]. Studies comparing contraction properties of lymphatics isolated from various regions within the rat lend credence to this hypothesis [23]. Common metrics to describe pump efficiency such as diastolic diameter, systolic diameter, and ejection fraction were found to be optimized to their physiologic locations. Vessels isolated from the lipoprotein-rich mesentery exhibited the strongest contractions compared to vessels from the thoracic duct. A conclusion we draw from this study is that the baseline vessel response to force is particular to the mechanical environment from which they are isolated. This mechanical environment is influenced by a variety of extrinsic mechanisms, including lymph formation, muscle contraction, pulsation of adjacent arteries, and gravitational forces [23]. Mesenteric vessels continued to pump even in the presence of enhanced shear while thoracic duct contraction frequency was completely abolished by shear stress. One possible explanation for this being that it is desirable for the mesenteric lymphatics to continue to pump during the elevated levels in viscosity and shear that would be experienced by the vessel during post-prandial lipid absorption.

Current evidence suggests that in addition to pump function, lymphatics can actively alter their permeability in response to changes in either luminal or transmural flow $[65,66]$. Increasing amounts of shear stress, such as those that might be seen downstream in collecting lymphatics upon a localized increase in lymph formation, decreases permeability through Rac1 induction of actin filament rearrangement [65]. Conversely, increases in transmural flow (i.e. flow through the junctions into the lymphatic) have the opposite effect, increasing permeability [66] which is likely to facilitate uptake and immune cell trafficking into the lymphatic. This differential response would often be desirable in situations of inflammation, for example, where one would expect localized increases in blood capillary permeability to cytokines such as TNF-alpha. Enhanced permeability in initial lymphatics would facilitate greater drainage of fluid and protein from the interstitium by the initial lymphatics, while downstream lymphatics would decrease their permeability as a result of elevated luminal fluid shear stress.

Comparative studies of rat mesenteric lymphatic vessels and venules suggest a similar permeability to albumin providing a role for lymphatics to participate in solute exchange under certain physiologic conditions [67]. This is particularly relevant in lymphedema where lymphatics are under higher transmural pressure [54] and thus the summation of forces on the vessel might favor albumin flux out of the vessel and into the interstitium. This study did assume that the reflection coefficient in collecting lymphatics for albumin is the same as it is in venules. While such an assumption is certainly reasonable given the shared lineage of the two vessel types, further studies are warranted to establish the role of protein flux out of the lymphatics in the etiology of diseases such as lymphedema.

\section{Modeling Lymphatic Biomechanics}

In addition to experimental approaches, mathematical models of lymphatic flow are being employed to characterize lymphatic function. In fact, mathematical modeling of the lymphatic system was one of the first tasks taken on by engineers working in lymphatic research and early work in this area has been reviewed by Reddy [68]. There have been numerous advances in computational capabilities since, as well as a significant increase in experimental data on lymphatic biomechanics as mentioned in the previous section. As a result of this, several new models have been proposed recently and have been compared 
against experimental data. A comprehensive model of lymphatic physiology, that could predict functional changes throughout the lymphatic system in response to a local change in load would be particularly useful in understanding the progression of lymphedema, the primary disease associated with lymphatic failure. While a lymphatic network model that takes into account individual vessel mechanics and has substantial predictive capability is still in the distant future, the building blocks have recently been put into place for implementing such an approach. In this section, we discuss lymphatic modeling in the context of three areas: initial lymphatic uptake, collecting lymphatic contractility, and vessel networks.

\section{Modeling initial lymphatic uptake}

Within the past decade, significant efforts have been made to describe the mechanism of uptake into the initial lymphatics. Initial lymphatics are open-ended structures comprised of endothelial cells forming capillaries that are approximately $10-30 \mu \mathrm{M}$ wide and 1 cell thick. The cells of the vessel integrate with one another to form specialized overlapping endothelial junctions, which serve as unidirectional valves. Additionally, anchoring proteins surround the structure providing external rigidity and support and pulling the junctions open when the local tissue expands. Experiments with fluorescently labeled microparticles were used to investigate the mechanisms of fluid entry into the initial lymphatics and their ability to prevent backflow after the vessel is filled with fluid. Fluorescent microparticles injected into the surrounding interstitium, were taken into the initial lymphatic, and remained there even when a negative pressure gradient was experimentally applied in an attempt to aspirate them. [35]. This suggests the existence of a unidirectional valve system in the initial lymphatic that responds to pressure gradients.

Further molecular characterization revealed tight intercellular junctions present in the indicative "oak leaf" pattern of overlapping endothelial cells, supported by localized expression of vascular endothelial (VE)-Cadherin formations described as "buttons" [36]. This is in direct contrast to the collecting lymphatics, where more striated patterns of VECadherin are observed forming "zipper" junctions. Interestingly, these specialized junctions did not appear along the tips of sprouting vessels, suggesting that the junctional "button" morphology is acquired after the development of the initial lymphatic [36].

Combining molecular characterization with simulation has allowed researchers to independently control experiment parameters in a manner previously not possible. Simulation of lymph formation through the initial lymphatic structures suggest that opportunistic fluctuations in interstitial pressure produce a resulting suction force. [69]. The flow through the intercellular junctions has been modeled using Equation 1

$$
Q_{\mathrm{tl}}=N_{\mathrm{L}} K_{\mathrm{L}} \int_{0}^{L}\left(P_{\text {in }}-P\right) d z
$$

where $Q_{\mathrm{tl}}$ is the flow into the initial lymphatics, $N_{\mathrm{L}}$ is number of initial lymphatics, $K_{\mathrm{L}}$ is the hydraulic conductivity per unit length, and $P_{\text {in }}$ and $P$ define the interstitial and luminal pressures respectively. The flow resistance of the intercellular junctions in this instance is reflected in the hydraulic conductivity term $K_{\mathrm{L}}$ for one junction which is explicitly defined in Equation 2,

$$
K_{\mathrm{L}}=\frac{q_{\text {pore }}}{\Delta P_{\text {pore }}}=\frac{\pi\left(R_{\text {pore }}\right)^{-1}}{8 \mu L_{\text {pore }}}
$$


where $q_{\text {pore }}$ is flow-rate through the pore, $\Delta P_{\text {pore }}$ is essentially the transmural pressure, $R_{\text {pore }}$ is pore radius, and $L_{\text {pore }}$ is the length of the pore. Using these equations to describe flow into the initial lymphatics revealed that at a length, $L$, which is $6-8$ times the vessel radius, $r$, the vessel no longer experiences suction as a result of the afferent collecting lymphatic. This implies that increasing the length of a initial lymphatic does not necessarily result in a flow volume increase. Furthermore, simulation suggests that lymphatic vessels that exhibit higher levels of anchoring proteins will correspondingly produce higher flow rates. These findings are particularly relevant to researchers utilizing lymphangiogenic approaches for treating lymphedema, as the number of LECs one can grow in a tissue does not necessarily correspond to increased lymphatic transport, but rather vessel length, morphology, and the local tissue support are all optimized for proper lymph drainage.

Modeling the flow into the initial lymphatics has evolved to consider the dynamic nature of overlapping endothelial cell junctions [70]. The primary valve system can be modeled as a beam with a deflection, $w(x)$, for a given moment. The magnitude of the applied moment, and subsequent beam deflection, is the result of negative pressures experienced during the contractile phase of the downstream collecting lymphatics as described in Equation 3,

$$
\frac{d^{4} w(x)}{d x^{4}}=\frac{1}{E I} \Delta P(x)
$$

where $W(x)$ is the deflection of the neighboring overlapping endothelial cell, $E$ is Young's modulus, $I$ is the moment of inertia, and $\Delta P(x)$ is the transendothelial pressure. Modeling the endothelial junction as a deflected beam suggests that the intraluminal pressure drops as the flow rate increases exponentially [70].

The deflected beam model has been further developed using computational fluid dynamics (CFD) for comparison purposes [71]. The computational model provides the advantages of performing sensitivity analysis for multiple parameters in 2 dimensions. The computational model was generated from a form of the Navier-Stokes equation to describe flow through a porous medium,

$$
\mu / k \mathrm{q}=-\nabla p+\mu_{e} \nabla^{2} \mathrm{q}
$$

where, $\mu$ is the bulk viscosity, $k$ is the permeability, $\mathrm{q}$ is the bulk parameter, $p$ is the pressure, and $\mu_{e}$ is the effective viscosity. Equation 4 is the basis for the computational model comparison to Equation 3. Computational model results revealed that the porosity value influenced the maximum cell deflection by a factor of 10 . Additionally, the model confirmed that during back-flow conditions, the resistance to flow approaches infinity, which agrees with experimental results [35].

\section{Modeling collecting lymphatic contractility}

The collecting lymphatics are the terminal point for the initial lymphatics and are lined with smooth muscle that propels lymph through the vessel. As discussed earlier, the pumping action and the resulting flow rate is greatly influenced by the mechanical forces subjected on the vessel. While extrinsic factors such as skeletal muscle contraction, vasomotion of the surrounding vasculature, and movement of the diaphragm are noted to promote lymph flow, most models published to date have focused on the instrinsic pumping of the lymphatic vessel as the primary mechanism promoting lymphatic flow. Modeling the the biomechanics of this process has a number of advantages for understanding the effects of lymphedema and the characteristic build up of interstitial fluid pressure on vessel function. Additionally, an 
accurate model of the fluid mechanics is necessary to estimate WSS from flow. Dimensional analysis of axisymetric Navier-Stokes flow, when coupled with experimental data from in vivo velocity and diameter measurements, demonstrated that Poiseuille flow is a reasonable approximation for the velocity distribution within the vessel, provided that fluid velocity is much greater than the wall velocity and velocity measurements are made far enough downstream from the lymphatic valves [37]. Fortunately these stipulations hold true for most of the contraction cycle and thus this approach was useful for generating the first estimates of WSS in lymphatic vessels in vivo. The assumption of Poiseuille flow was further verified by comparing it to a more complex model (Equations 5-6) of a radially expanding and contracting lymphangion [72],

$$
\begin{gathered}
\mathbf{T}=\mu\left[\nabla \mathbf{v}+\nabla \mathbf{v}^{\mathrm{T}}-\frac{\mathbf{2}}{\mathbf{3}}(\nabla \cdot \mathbf{v}) \mathbf{I}\right] \\
\overrightarrow{\tau_{w}}=-\mathbf{T} \cdot \frac{\mathbf{a}}{|\mathbf{a}|}
\end{gathered}
$$

where $\mathbf{a}$ is the axial face area vector, $\mathbf{v}$ is the velocity vector, $\mu$ is the dynamic viscosity and $\mathbf{T}$ is the stress tensor. Using CFD and incorporating physiologic waveforms into this model demonstrated that the assumption of Poiseuille introduces error of around 4\%, making this simplification reasonable for estimating WSS for most of the contraction cycle, as the experimental velocity measurements are usually the larger source of error.

Other deviations of Poiseuille flow have been published that incorporate the elasticity of the vessel wall rather than treating it as a rigid tube $[61,73,74]$. Models of the left ventricle have modeled the dynamic elasticity, $E_{d}$, as a term to describe the relationship between the chamber pressure, $P_{t}$, and volume, $V$, as a function of time [75,76]. Using a similar approach, Equation 7 provides a means to quantify contractility in both pre and post-loading conditions in a contracting lymphatic.

$$
E_{d}(t)=\frac{P_{t}(t)}{V(t)-V_{0}}
$$

Equation 7 has been used with a previously derived relationship to describe blood flow through an artery:

$$
\Delta P=\frac{8 \mu L}{\pi r^{4}} Q+\frac{\rho L}{\pi r^{2}} \frac{d Q}{d t}
$$

where $\Delta P$ is the pressure gradient across a tube length $L, \mu$, is the lymph viscosity, $r$ is the vessel radius, and $\rho$ is the lymph density. Applying Equations 7-8 in conjunction with conservation of mass and comparing to experimental data from excised bovine vessels reveals that collecting lymphatics are sensitive to a pressure gradient and in the presence of flow the vessel seeks to minimize contractility. In addition, the induced pressure gradient effectively opened the inter-lymphangion valves when pressures exceeded $0.25 \mathrm{mmHg}$ [61]. More recently, Davis has shown that distention of the vessel modulates leaflet stiffness and consequently the resulting pressure gradient needed to open and close the lymphatic valve [77]. This is a very exciting study and should provide future insight into the role of distention induced valve failure in lymphedema. 
The results of both the theoretical and experimental models suggest a pressure sensitivity that guides the bimodal pump/conduit behavior of a vessel in vivo. Subsequent to this, Quick introduced an equivalent circuit, first order approximation of their previous model and demonstrated that this drastic simplification produced remarkably similar results when compared to the previous approach [78]. Macdonald built upon the studies of Reddy and Quick by incorporating a dampening and a longitudinal bending term in the equation describing the vessel wall behavior. This model was then fit to experimental data to elucidate the mechanical properties of the lymphatic wall. They too observed a pump/ conduit behavior in their model, where in certain situations flow would actually be increased by minimizing vessel contraction. Even though increasing the complexity of these models has produced strikingly similar results when compared to much simpler approaches, we anticipate these more complex models that capture the mechanical properties of the vessel wall to be extremely useful in understanding lymphatic behavior in disease conditions.

\section{Lymphatic network modeling}

In addition to understanding the contribution of an individual lymphatic vessel in determining flow, lymphatic system models that incorporate network analysis provide an opportunity for the development of a prognostic tool. Predictive capabilities would be particularly important for modeling lymphatic disease and/or vessel removal (as is often performed in a mastectomy). A variety of methods have been published, with the most basic approach being to lump lymphatic drainage into a single parameter in order to explain its interplay with the circulation in regulating fluid balance [79]. Applying algebraic formulations in the context of balance point analysis provides a straightforward method to quantify individual contributions from the microvasculature, interstitial pressure, and lymphatic function. Balance point analysis suggests that steady state interstitial pressure balance can be modeled by multiplying lymphatic resistance by a microvascular filtration coefficient [79]. Additionally, interstitial pressure is highly directed by capillary conditions more so than by lymphatic outlet pressure. Balance point analysis provides an easily implemented means for calculating interstitial steady state conditions [79].

Others have built upon the previously mentioned models of individual vessels to observe their ability to coordinate the transport of fluid by considering them as a chain of contracting elements [74,80-82]. Lymphatic network modeling suggests a balance may be achieved between contraction frequency and amplitude to provide an optimal flow rate and that the length of each lymphangion within the network is designed to promote flow [81]. The assumptions that form the basis of this model were that: 1)The maximum shear stress is calculated using the end diastolic radius. 2) Contraction frequencies are assumed to be constant, which may be appropriate for a short analysis time. 3) The lymphatic is modeled to act as a pump at all times. 4) The modeled lymphatic system is assumed to be symmetric through each bifurcation point. 5) Each lymphatic vessel seeks to maintain a similar WSS. Using these assumptions, the lymphatic system can be modeled as a fractal system showing scale independent self similarity. Applying fractal analysis for calculating the optimal length/radii ratio reveals a value of 10 and a bifurcation number of 21 . While the assumptions that went into the model are often invalid, these values are remarkably close to reported bovine lymphatics.

Computational methods have also been used to simulate collapsible contracting lymphangions with progressive valve closure [82]. Considering the flow through a lymphangion as similar to current flow through a diode with constant resistance, the flow through a segment, $Q_{i+1}$ can be approximated by Equation 9, 


$$
Q_{i+1}=Q_{i}-\frac{\pi}{2} D_{i} L \frac{d D_{i}}{d t}
$$

where $Q_{i}$ is the flow into the segment, $D_{i}$ is a time dependent diameter, and $L$ is the length of the lymphangion. Additionally, each segment assumes fully develeoped Poiseuille flow and conservation of momentum seen below,

$$
\frac{p_{i 1}-p_{i 2}}{L}=\frac{64 \mu\left(Q_{i}+Q_{i+1}\right)}{\pi D_{i}^{4}}
$$

where $p_{i 1}$ and $p_{i 2}$ are the pressures at the entrance and exit of the lymphangion respectively, $t$ is time, and $\mu$ is the dynamic viscosity. The vessel wall constitutive relation is modeled having both passive and active components in Equation 11,

$$
\left.p_{t m}=\frac{p_{i 1}+p_{i 2}}{2}-p_{e}=P_{d i}\left(e^{D_{i} / D_{d i}}-\left(D_{d i} / D_{i}\right)^{3}\right)\right)+\frac{M}{D_{i}}\left[1-\cos \left[2 \pi f\left(t-t_{o i}\right)\right]\right]
$$

where $p_{t m}$ is the transmural pressure, $f$ is the frequency, and $M$ is a parameter to describe contraction strength and having the dimensions of force per unit length. The first term describes the passive wall stiffening at $+p_{t m}$ with the exponential function and diminishing compliance at $p_{t m}$ with the cubic function. The valve resistance can be modeled as two sigmoidal functions describing resistance at a small pressure drop $p_{\text {open }}$ and at detrimental large pressures that induce back-flow $p_{\text {fail }}$,

$$
R_{V}=R_{V \min }+R_{V \max }\left(\frac{1}{1+\exp \left(s_{\text {open }}\left(\Delta p-p_{\text {open }}\right)\right)}+\frac{1}{1+\exp \left(-s_{\text {fail }}\left(\Delta p-p_{\text {fail }}\right)\right)}-1\right)
$$

where $\Delta P=p_{12}-p_{21}$. Implementing Equations 9-12 in a non-linear minimum finding ordinary differential equation (ODE) solver yields unique solutions for the flow, pressure, and resistance in each discrete lymphangion. This model is very flexible in the sense that it easily allows for multiple lymphangion simulations in series. This approach suggests that the effect of additional lymphangions to the contribution to outflow, $\bar{Q}$ may be minimal. Also, $\bar{Q}$ is maximized when lymphangion contractions are asynchronous.

Modeling vessel dynamics is furthering our understanding of the physical parameters that influence the flow output of lymphatics and will help guide experiments as we seek to understand the intrinsic mechanisms that ensure proper lymphatic function. However, in their current state, the predictive power of these models is limited, particularly as it relates to disease, and the more detailed models of individual vessel solid mechanics have not yet been incorporate into the larger lymphatic network models. Continuing to refine these models to better represent the in vivo biomechanics will provide an effective means to explore the pressure/flow relationship in lymphatics.

\section{Interstitial Flow}

Since the lymphatics are constantly removing excess fluid and protein from the interstitium, there necessarily exists a slow interstitial flow that is directed towards the nearest draining lymphatic vessel. While the physics of interstitial flow is not the focus of this review (we refer the reader to an excellent review on this topic [83]), it has been shown to play an important role in development and modulation of essential lymphatic functions in both 
health and disease, which we feel are important to mention here. Interstitial flow was first recognized as a guide for lymphangiogenesis in a mouse tail lymphatic regeneration model [84]. By altering the geometry of the wound to manipulate the path interstitial fluid is forced to take through the tail, Boardman showed that lymphangiogenesis was impaired when interstitial flow was redirected around the wound compared to the case when interstitial flow went through it. Subsequent studies demonstrated that this regeneration was facilitated by interstitial flow through enhanced transport of both endogenous matrix metalloproteinases and lymphangiogenic growth factors through the regenerating region [85]. Interstitial levels of flow (less than $10 \mu \mathrm{m} / \mathrm{s}$ ) have also been shown to be important in vessel growth and development in vitro, producing a differential response between blood and lymphatic endothelial cells [32], and promoting sprouting and lumen formation in part through gradient amplification of soluble and matrix-binding growth factors [86,33]. By coupling this biophysical phenomenon with engineered matrix-binding, fusion proteins, Helm was able to demonstrate enhanced lymphatic growth in a 3D tissue-engineered construct [31].

It is likely that lymphatic tissue-engineering will require similar approaches if we are to realize a functional, tissue-engineered lymphatic network for treating lymphedema, as it is becoming apparent that tissue constructs grown under conditions resembling their native biophysical environment are better suited to handle the physiologic functions required of them in vivo [87]. In addition to lymphangiogenesis, interstitial flow enhances secretion and subsequent gradient formation of chemokines in order to facilitate immune cell trafficking towards lymph nodes via the nearest draining lymphatic vessel (termed autologous chemotaxis) $[88,89]$. Interestingly, certain tumor cells have hijacked immune cells to navigate towards lymphatics using a very similar autologous chemotaxis mechanism $[90,66]$. How pathological changes to interstitial flow in conditions such as lymphedema enhance disease progression currently remains unknown, although numerous lines of evidence suggest such connections exist [91].

\section{Biomechanics in lymphatic disease}

One of the least studied areas in lymphatic biomechanics, and perhaps one of the most important, is the alteration of biomechanics in lymphatic disease. Given that lymphatics alter their function in response to changes in mechanical load (e.g. stretch and wall shear stress), it is essential that we understand how various lymphatic diseases and pathologies alter the mechanical environment experienced by the vessels. This is particularly true in lymphedema, where lymphatic transmural pressure is likely elevated [54], and chronic lymph stasis results in an accumulation of collagen and adipocytes, which can drastically alter the mechanical environment of the tissue surrounding the initial lymphatic [13]. Initial lymphatics can be quite responsive to these local environmental changes as myofibroblasts create tissue tension in wound healing that serves as a mechanical cue to direct neovascularization from pre-existing vessels [92]. While such a response is beneficial in a normal wound healing situation, how these mechanisms are implemented in a state of unwanted tissue remodeling like that which occurs in lymphedema remains unknown. Clinically, lymphedema almost always presents itself in the extremities, where the mechanical work needed to be done to transport the fluid back into the blood is the greatest. In fact, it is essentially impossible to generate a secondary lymphedema model in a mouse that is as severe as that seen in humans, likely due to the much smaller distances that have to be overcome by the lymphatic system in mice. In addition to secondary lymphedema, patients surviving from congenital heart defects, such as those that have a Fontan circulation, often present themselves with elevated venous pressure and lymphatic dysfunction [93]. All of these varying lines of evidence indicate that mechanics is essential to disease onset and/or progression. 
Lymphedema is often associated with inflammation and given that chronic inflammation has been shown to reduce the effectiveness of the primary lymphatic valves to prevent backflow [94], lymphedema-induced inflammation would tend to lead to more fluid accumulation, establishing a positive-feedback cycle that might be difficult to break. How these different mechanical and inflammatory changes in the interstitium alter the shear stress and tensile forces in downstream collecting lymphatics and how the vessels adapt their function to sustained changes in their microenvironment remains unknown. An animal model of secondary lymphedema has shown morphological changes in lymphatic vessels in which the vessels increase in diameter in part through an up-regulation in vascular endothelial growth factor C (VEGF-C) [95]. While VEGF-C is primarily involved in lymphangiogenesis, one study showed that exogenous VEGF-C increases lymphatic contractility through its receptor (VEGFR-3) located on LECs [96]. Whether endogenous levels of VEGF-C can modulate lymphatic contraction, or whether VEGF-C release is regulated through mechanotransduction pathways remains unknown. It is striking how little is known about the biomechanics of lymphatics in lymphedema, as the primary treatments in practice today for patients suffering from lymphedema are mechanical in nature - compression garments and massage. Coupling these approaches with a more complete understanding of lymphatic biomechanics will be crucial to improving the efficacy of lymphedema treatment.

\section{Conclusions}

The lymphatics have evolved a highly sensitive functional response to the widely varying loads and demands placed on the vessel in vivo, which we have only just begun to understand. Advances in lymphatic imaging, experimental approaches, and tissue engineered constructs of the lymphatic vasculature have made significant contributions to our understanding of lymphatic physiology in both health and disease. Using these approaches to probe the functional response of lymphatics to alterations in mechanical load, both in the short term and in the long term, will greatly enhance our understanding of the progression of lymphatic diseases. Accurate quantification of the mechanical microenvironment in disease progression in vivo will better inform in vitro experimental design. Lastly, incorporating these observations into computational models of the lymphatics that have predictive capability, on both the individual vessel and system levels, will greatly improve the efficacy of treatments for those suffering from lymphedema, opening the door for a personalized medicine approach in lymphedema therapy.

\section{Acknowledgments}

This work was supported in part through NIH R00HL091133.

\section{References}

1. Randolph G, Angeli V, Swartz MA. Dendritic-cell trafficking to lymph nodes through lymphatic vessels. Nature Reviews Immunology. 2005 Jul; 5(8):617-628.

2. Dixon JB. Lymphatic lipid transport: sewer or subway? Trends in Endocrinology and Metabolism: TEM. 2010 Jun; 21(8):480-487.

3. Lund AW, Swartz MA. Role of lymphatic vessels in tumor immunity: passive conduits or active participants? Journal of Mammary Gland Biology and Neoplasia. 2010 Sep; 15(3):341-352. [PubMed: 20835756]

4. Tammela T, Alitalo K. Lymphangiogenesis: Molecular mechanisms and future promise. Cell. 2010 Feb; 140(4):460-476. [PubMed: 20178740]

5. Levick JR, Michel CC. Microvascular fluid exchange and the revised Starling principle. Cardiovascular Research. 2010 Jul; 87(2):198-210. [PubMed: 20200043] 
6. Swartz MA. The physiology of the lymphatic system. Advanced Drug Delivery Reviews. 2001 Aug; 50(1-2):3-20. [PubMed: 11489331]

7. Jeltsch M, Kaipainen A, Joukov V, Meng X, Lakso M, Rauvala H, et al. Hyperplasia of lymphatic vessels in VEGF-C transgenic mice. Science. 1997 May; 276(5317):1423-1425. [PubMed: 9162011]

8. Banerji S, Ni J, Wang SX, Clasper S, Su J, Tammi R, et al. LYVE-1, a new homologue of the CD44 glycoprotein, is a lymph-specific receptor for hyaluronan. The Journal of Cell Biology. 1999 Feb; 144(4):789-801. [PubMed: 10037799]

9. Proulx ST, Luciani P, Derzsi S, Rinderknecht M, Mumprecht V, Leroux JC, et al. Quantitative Imaging of Lymphatic Function with Liposomal Indocyanine Green. Cancer Research. 2010 Sep; 70(18):7053-7062. [PubMed: 20823159]

10. Swartz MA, Berk D, Jain R. Transport in lymphatic capillaries.1. Macroscopic measurements using residence time distribution theory. American Journal Of Physiology. 1995 Dec; 39(1):H324H329.

11. Berk DA, Swartz MA, Leu AJ, Jain RK. Transport in lymphatic capillaries. II. Microscopic velocity measurement with fluorescence photobleaching. The American Journal of Physiology. 1996 Jan.270:H330-7. [PubMed: 8769769]

12. Swartz MA, Kaipainen A, Netti P, Brekken C, Boucher Y, Grodzinsky A, et al. Mechanics of interstitial-lymphatic fluid transport: theoretical foundation and experimental validation. Journal of Biomechanics. 1999 Nov; 32(12):1297-1307. [PubMed: 10569708]

13. Rutkowski JM, Markhus CE, Gyenge CC, Alitalo K, Wiig H, Swartz MA. Dermal collagen and lipid deposition correlate with tissue swelling and hydraulic conductivity in murine primary lymphedema. The American Journal of Pathology. 2010 Mar; 176(3):1122-1129. [PubMed: 20110415]

14. Lim HY, Rutkowski JM, Helft J, Reddy ST, Swartz MA, Randolph GJ, et al. Hypercholesterolemic mice exhibit lymphatic vessel dysfunction and degeneration. The American Journal of Pathology. 2009 Sep; 175(3):1328-1337. [PubMed: 19679879]

15. Dixon JB, Zawieja DC, Gashev AA, Cote G. Measuring microlymphatic flow using fast video microscopy. Journal of Biomedical Optics. 2005; 10(6):064016. [PubMed: 16409081]

16. Dixon JB, Gashev AA, Zawieja DC, Moore JE Jr, Cote G. Image correlation algorithm for measuring lymphocyte velocity and diameter changes in contracting microlymphatics. Annals of Biomedical Engineering. 2007; 35(3):387-396. [PubMed: 17151922]

17. Sharma R, Wang W, Rasmussen J, Joshi A, Houston J, Adams K, et al. Quantitative imaging of lymph function. American Journal of Physiology: Heart and Circulatory Physiology. 2007 May; 292(6):H3109-18. [PubMed: 17307997]

18. Kwon S, Sevick-Muraca EM. Functional lymphatic imaging in tumor-bearing mice. Journal of Immunological Methods. $2010 \mathrm{Jul}$.

19. Sevick-Muraca EM, Sharma R, Rasmussen JC, Marshall MV, Wendt JA, Pham HQ, et al. Imaging of lymph flow in breast cancer patients after microdose administration of a near-infrared fluorophore: feasibility study. Radiology. 2008 Mar; 246(3):734-741. [PubMed: 18223125]

20. Unno N, Nishiyama M, Suzuki M, Yamamoto N, Inuzuka K, Sagara D, et al. Quantitative lymph imaging for assessment of lymph function using indocyanine green fluorescence lymphography. European Journal of Vascular and Endovascular Surgery. 2008 Jul; 36(2):230-236. [PubMed: 18534875]

21. Suzuki M, Unno N, Yamamoto N, Nishiyama M, Sagara D, Tanaka H, et al. Impaired lymphatic function recovered after great saphenous vein stripping in patients with varicose vein: venodynamic and lymphodynamic results. Journal of Vascular Surgery. 2009 Nov; 50(5):10851091. [PubMed: 19632804]

22. Gashev AA, Nagai T, Bridenbaugh EA. Indocyanine green and lymphatic imaging: current problems. Lymphat Res Biol. 2010 Jun; 8(2):127-130. [PubMed: 20583875]

23. Gashev AA, Davis MJ, Delp M, Zawieja DC. Regional variations of contractile activity in isolated rat lymphatics. Microcirculation (New York, NY: 1994). 2004 Aug; 11(6):477-492. 
24. Gashev AA, Davis MJ, Gasheva OY, Nepiushchikh ZV, Wang W, Dougherty P, et al. Methods for Lymphatic Vessel Culture and Gene Transfection. Microcirculation (New York, NY: 1994). 2009 Jan; 16(7):615-628.

25. Cooke C, Nanjee M, Stepanova I, Olszewski W, Miller N. Variations in lipid and apolipoprotein concentrations in human leg lymph: effects of posture and physical exercise. Atherosclerosis. 2004 Feb; 173(1):39-45. [PubMed: 15177122]

26. Nanjee M, Cooke C, Wong J, Hamilton R, Olszewski W, Miller N. Composition and ultrastructure of size subclasses of normal human peripheral lymph lipoproteins: quantification of cholesterol uptake by HDL in tissue fluids. Journal of Lipid Research. 2001 Mar; 42(4):639-648. [PubMed: 11290836]

27. Leak LV, Liotta LA, Krutzsch H, Jones M, Fusaro VA, Ross SJ, et al. Proteomic analysis of lymph. Proteomics. 2004 Mar; 4(3):753-765. [PubMed: 14997497]

28. Lautenschläger I, Dombrowsky H, Frerichs I, Kuchenbecker SC, Bade S, Schultz H, et al. A model of the isolated perfused rat small intestine. American Journal of Physiology-Gastrointestinal and Liver Physiology. 2010 Feb; 298(2):G304-13. [PubMed: 19910525]

29. Dixon JB, Raghunathan S, Swartz MA. A tissue-engineered model of the intestinal lacteal for evaluating lipid transport by lymphatics. Biotechnology and Bioengineering. 2009 Aug; 103(6): 1224-1235. [PubMed: 19396808]

30. Dixon JB. Mechanisms of chylomicron uptake into lacteals. Annals of the New York Academy of Sciences. 2010 Oct; 1207( Suppl 1):E52-7. [PubMed: 20961306]

31. Helm C, Zisch A, Swartz MA. Engineered blood and lymphatic capillaries in 3-D VEGF-fibrincollagen matrices with interstitial flow. Biotechnology and Bioengineering. 2007; 96(1):167-176. [PubMed: 17133613]

32. $\mathrm{Ng} \mathrm{C}$, Helm C, Swartz MA. Interstitial flow differentially stimulates blood and lymphatic endothelial cell morphogenesis in vitro. Microvascular Research. 2004 Oct; 68(3):258-264 [PubMed: 15501245]

33. Helm C, Fleury M, Zisch A, Boschetti F, Swartz MA. Synergy between interstitial flow and VEGF directs capillary morphogenesis in vitro through a gradient amplification mechanism. Proceedings of the National Academy of Sciences. 2005 Oct; 102(44):15779-15784.

34. Schmid-Schonbein G. Microlymphatics and lymph flow. Physiological Reviews. 1990 Sep; 70(4): 987-1028. [PubMed: 2217560]

35. Trzewik J, Mallipattu S, Artmann G, Delano F, Schmid-Schonbein G. Evidence for a second valve system in lymphatics: endothelial microvalves. The FASEB journal: official publication of the Federation of American Societies for Experimental Biology. 2001; 15(10):1711-1717.

36. Baluk P, Fuxe J, Hashizume H, Romano T, Lashnits E, Butz S, et al. Functionally specialized junctions between endothelial cells of lymphatic vessels. The Journal of Experimental Medicine. 2007 Oct; 204(10):2349-2362. [PubMed: 17846148]

37. Dixon JB, Moore JE Jr, Cote G, Gashev AA, Zawieja DC. Lymph flow, shear stress, and lymphocyte velocity in rat mesenteric prenodal lymphatics. Microcirculation (New York, NY: 1994). 2006; 13(7):597-610.

38. Muthuchamy M, Gashev AA, Boswell N, Dawson N, Zawieja DC. Molecular and functional analyses of the contractile apparatus in lymphatic muscle. The FASEB journal: official publication of the Federation of American Societies for Experimental Biology. 2003 Feb; 17(3):920-922.

39. Kvietys PR, Granger DN. Role of intestinal lymphatics in interstitial volume regulation and transmucosal water transport. Annals of the New York Academy of Sciences. 2010 Oct; 1207( Suppl 1):E29-43. [PubMed: 20961304]

40. Gashev AA, Davis MJ, Zawieja DC. Inhibition of the active lymph pump by flow in rat mesenteric lymphatics and thoracic duct. Journal of Physiology. 2002 Apr; 540(3):1023-1037. [PubMed: 11986387]

41. Hargens AR, Zweifach BW. Contractile stimuli in collecting lymph vessels. The American Journal of Physiology. 1977 Jul; 233(1):H57-65. [PubMed: 879337]

42. McHale NG, Roddie IC. The effect of transmural pressure on pumping activity in isolated bovine lymphatic vessels. J Physiol. 1976 Oct; 261(2):255-269. [PubMed: 988184] 
43. Benoit JN, Zawieja DC, Goodman AH, Granger HJ. Characterization of intact mesenteric lymphatic pump and its responsiveness to acute edemagenic stress. The American Journal of Physiology. 1989 Dec.257:H2059-69. [PubMed: 2603989]

44. von der Weid PY, Zawieja DC. Lymphatic smooth muscle: the motor unit of lymph drainage. The International Journal of Biochemistry and Cell Biology. 2004 Jul; 36(7):1147-1153.

45. Davis MJ, Davis AM, Lane MM, Ku CW, Gashev AA. Rate-sensitive contractile responses of lymphatic vessels to circumferential stretch. The Journal of Physiology. 2009 Jan.587:165-182. [PubMed: 19001046]

46. Zawieja DC, Davis K, Schuster R, Hinds W, Granger H. Distribution, Propagation, and Coordination of Contractile Activity in Lymphatics. American Journal Of Physiology. 1993 Mar; 264(4):H1283-H1291. [PubMed: 8476104]

47. McHale NG, Meharg MK. Co-ordination of pumping in isolated bovine lymphatic vessels. The Journal of Physiology. 1992 May.450:503-512. [PubMed: 1432715]

48. Akl TJ, Nepiyushchikh ZV, Gashev AA, Zawieja DC, Cot GL. Measuring contraction propagation and localizing pacemaker cells using high speed video microscopy. Journal of Biomedical Optics. 2011 Feb.16(2):026016. [PubMed: 21361700]

49. Shirasawa Y, Benoit JN. Stretch-induced calcium sensitization of rat lymphatic smooth muscle. American Journal of Physiology: Heart and Circulatory Physiology. 2003 Dec; 285(6):H2573-7. [PubMed: 12946938]

50. Von der Weid PY, Van Helden DF. Beta-adrenoceptor-mediated hyperpolarization in lymphatic smooth muscle of guinea pig mesentery. Am J Physiol. 1996 May.270:H1687-H1695. [PubMed: 8928875]

51. Toland HM, McCloskey KD, Thornbury KD, McHale NG, Hollywood MA. Ca(2+)-activated $\mathrm{Cl}(-)$ current in sheep lymphatic smooth muscle. American Journal of Physiology: Heart and Circulatory Physiology. 2000 Nov; 279(5):C1327-C1335.

52. Atchison DJ, Rodela H, Johnston MG. Intracellular calcium stores modulation in lymph vessels depends on wall stretch. Canadian journal of physiology and Pharmacology. 1998 Apr; 76(4):367372. [PubMed: 9795744]

53. Dougherty PJ, Davis MJ, Zawieja DC, Muthuchamy M. Calcium sensitivity and cooperativity of permeabilized rat mesenteric lymphatics. American Journal Of Physiology: Regulatory, Integrative, and Comparitive Physiology. 2008 May; 294(5):R1524-32.

54. Gretener SB, Läuchli S, Leu AJ, Koppensteiner R, Franzeck UK. Effect of venous and lymphatic congestion on lymph capillary pressure of the skin in healthy volunteers and patients with lymph edema. Journal Of Vascular Research. 2000; 37(1):61-67. [PubMed: 10720887]

55. Gasheva OY, Zawieja DC, Gashev AA. Contraction-initiated NO-dependent lymphatic relaxation: a self-regulatory mechanism in rat thoracic duct. The Journal of Physiology. 2006 Sep.575:821832. [PubMed: 16809357]

56. Davies P. Flow-mediated endothelial mechanotransduction. Physiological Reviews. 1995 Jun; 75(3):519-560. [PubMed: 7624393]

57. Kuo L, Davis MJ, Chilian WM. Endothelium-dependent, flow-induced dilation of isolated coronary arterioles. American Journal Of Physiology. 1990 Oct.259:H1063-70. [PubMed: 2221113]

58. Kawai Y, Yokoyama Y, Kaidoh M, Ohhashi T. Shear stress-induced ATP-mediated endothelial constitutive nitric oxide synthase expression in human lymphatic endothelial cells. American Journal Of Physiology: Cell Physiology. 2010 Mar; 298(3):C647-55. [PubMed: 20042732]

59. Lipowsky H. Microvascular rheology and hemodynamics. Microcirculation (New York, NY: 1994). 2004 Dec; 12(1):5-15. 904CI Times Cited:2 Cited References Count:77.

60. Koller A, Mizuno R, Kaley G. Flow reduces the amplitude and increases the frequency of lymphatic vasomotion: role of endothelial prostanoids. American Journal Of Physiology. 1999 Nov; 277(6):R1683-R1689. [PubMed: 10600914]

61. Quick CM, Venugopal AM, Gashev AA, Zawieja DC, Stewart RH. Intrinsic pump-conduit behavior of lymphangions. American Journal Of Physiology: Regulatory, Integrative, and Comparitive Physiology. 2007 Apr; 292(4):R1510-8. 
62. Quick CM, Ngo BL, Venugopal AM, Stewart RH. Lymphatic pump-conduit duality: contraction of postnodal lymphatic vessels inhibits passive flow. American Journal of Physiology: Heart and Circulatory Physiology. 2009 Mar; 296(3):H662-8. [PubMed: 19122167]

63. Zhang RZ, Gashev AA, Zawieja DC, Davis MJ. Length-tension relationships of small arteries, veins, and lymphatics from the rat mesenteric microcirculation. American Journal of Physiology: Heart and Circulatory Physiology. 2007 Apr; 292(4):H1943-52. [PubMed: 17172274]

64. Humphrey JD. Vascular adaptation and mechanical homeostasis at tissue, cellular, and sub-cellular levels. Cell Biochemistry and Biophysics. 2008 Jan; 50(2):53-78. [PubMed: 18209957]

65. Breslin JW, Kurtz KM. Lymphatic endothelial cells adapt their barrier function in response to changes in shear stress. Lymphatic Research and Biology. 2009 Dec; 7(4):229-237. [PubMed: 20143922]

66. Miteva DO, Rutkowski JM, Dixon JB, Kilarski W, Shields JD, Swartz MA. Transmural flow modulates cell and fluid transport functions of lymphatic endothelium. Circulation Research. 2010 Mar; 106(5):920-931. [PubMed: 20133901]

67. Scallan JP, Huxley VH. In vivo determination of collecting lymphatic vessel permeability to albumin: a role for lymphatics in exchange. J Physiol. 2010 Jan; 588(Pt 1):243-254. [PubMed: 19917564]

68. Reddy N. Lymph Circulation - Physiology, Pharmacology, and Biomechanics. Critical Reviews in Biomedical Engineering. 1986; 14(1):45-91. [PubMed: 3524994]

69. Reddy NP, Patel K. A mathematical model of flow through the terminal lymphatics. Medical Engineering and Physics. 1995 Mar; 17(2):134-140. [PubMed: 7735643]

70. Mendoza E, Schmid-Schonbein G. A model for mechanics of primary lymphatic valves. Journal Of Biomechanical Engineering: Transactions Of The ASME. 2003 Jan; 125(3):407-414.

71. Galie P, Spilker RL. A two-dimensional computational model of lymph transport across primary lymphatic valves. Journal Of Biomechanical Engineering. 2009 Nov.131(11):111004. [PubMed: 20353255]

72. Rahbar E, Moore JE. A model of a radially expanding and contracting lymphangion. Journal of Biomechanics. 2011 Mar.

73. Reddy NP, Kesavan SK. Low Reynolds number liquid propulsion in contracting tubular segments connected through valves. Mathematical and Computer Modelling. 1989; 12(7):839_ 844.10.1016/0895-7177(89)90138-6

74. Macdonald A, Arkill K, Tabor G, McHale N, Winlove C. Modeling flow in collecting lymphatic vessels: one-dimensional flow through a series of contractile elements. American Journal of Physiology: Heart and Circulatory Physiology. 2008 Jun; 295(1):H305-13. [PubMed: 18487438]

75. Suga H, Sagawa K, Shoukas AA. Load independence of the instantaneous pressure-volume ratio of the canine left ventricle and effects of epinephrine and heart rate on the ratio. Circulation Research. 1973 Mar; 32(3):314-322. [PubMed: 4691336]

76. Suga H, Sagawa K. Instantaneous pressure-volume relationships and their ratio in the excised, supported canine left ventricle. Circulation Research. 1974 Jul; 35(1):117-126. [PubMed: 4841253]

77. Davis MJ, Rahbar E, Gashev AA, Zawieja DC, Moore JE. Determinants of valve gating in collecting lymphatic vessels from rat mesentery. Am J Physiol Heart Circ Physiol. 2011 Apr.

78. Quick CM, Venugopal AM, Dongaonkar RM, Laine GA, Stewart RH. First-order approximation for the pressure-flow relationship of spontaneously contracting lymphangions. American Journal of Physiology: Heart and Circulatory Physiology. 2008 Jan; 294(5):H2144-H2149. [PubMed: 18326809]

79. Dongaonkar RM, Laine GA, Stewart RH, Quick CM. Balance point characterization of interstitial fluid volume regulation. American Journal Of Physiology: Regulatory, Integrative, and Comparitive Physiology. 2009 Jul; 297(1):R6-16.

80. Venugopal AM, Stewart RH, Laine GA, Dongaonkar RM, Quick CM. Lymphangion coordination minimally affects mean flow in lymphatic vessels. American Journal of Physiology: Heart and Circulatory Physiology. 2007 Aug; 293(2):H1183-9. [PubMed: 17468331] 
81. Venugopal AM, Quick CM, Laine GA, Stewart RH. Optimal postnodal lymphatic network structure that maximizes active propulsion of lymph. American Journal of Physiology: Heart and Circulatory Physiology. 2009 Feb; 296(2):H303-9. [PubMed: 19028799]

82. Bertram CD, Macaskill C, Moore JE. Simulation of a chain of collapsible contracting lymphangions with progressive valve closure. Journal Of Biomechanical Engineering. 2011 Jan. 133(1):011008. [PubMed: 21186898]

83. Swartz MA, Fleury ME. Interstitial flow and its effects in soft tissues. Annual Review Of Biomedical Engineering. 2007 Jan.9:229-256.

84. Boardman KC, Swartz MA. Interstitial flow as a guide for lymphangiogenesis. Circulation Research. 2003 Apr; 92(7):801-808. [PubMed: 12623882]

85. Goldman J, Conley KA, Raehl A, Bondy DM, Pytowski B, Swartz MA, et al. Regulation of lymphatic capillary regeneration by interstitial flow in skin. American Journal of Physiology: Heart and Circulatory Physiology. 2007 May; 292(5):H2176-83. [PubMed: 17189348]

86. Fleury M, Boardman K, Swartz MA. Autologous morphogen gradients by subtle interstitial flow and matrix interactions. Biophysical Journal. 2006 Jun; 91(1):113-121. [PubMed: 16603487]

87. Zaucha MT, Raykin J, Wan W, Gauvin R, Auger FA, Germain L, et al. A novel cylindrical biaxial computer-controlled bioreactor and biomechanical testing device for vascular tissue engineering. Tissue Engineering: Part A. 2009 Nov; 15(11):3331-3340. [PubMed: 19385725]

88. Shields J, Fleury M, Yong C, Tomei A, Randolph G, Swartz MA. Autologous Chemotaxis as a Mechanism of Tumor Cell Homing to Lymphatics via Interstitial Flow and Autocrine CCR7 Signaling. Cancer Cell. 2007; 11(6):526-538. [PubMed: 17560334]

89. Shieh AC, Swartz MA. Regulation of tumor invasion by interstitial fluid flow. Physical Biology. 2011 Feb.8(1):015012. [PubMed: 21301060]

90. Swartz MA, Hubbell JA, Reddy S. Lymphatic drainage function and its immunological implications: From dendritic cell homing to vaccine design. Seminars in Immunology. 2008 Apr; 20(2):147-156. [PubMed: 18201895]

91. Bates DO. An interstitial hypothesis for breast cancer related lymphoedema. Pathophysiology: the official journal of the International Society for Pathophysiology/ISP. 2010 Sep; 17(4):289-294. [PubMed: 19963358]

92. Kilarski WW, Samolov B, Petersson L, Kvanta A, Gerwins P. Biomechanical regulation of blood vessel growth during tissue vascularization. Nature Medicine. 2009 Jun; 15(6):657-664.

93. Gewillig M. The Fontan circulation. Heart. 2005 Jun; 91(6):839-846. [PubMed: 15894794]

94. Lynch PM, Delano FA, Schmid-Schönbein GW. The primary valves in the initial lymphatics during inflammation. Lymphatic Research and Biology. 2007 Jan; 5(1):3-10. [PubMed: 17508898]

95. Rutkowski JM, Moya M, Johannes J, Goldman J, Swartz MA. Secondary lymphedema in the mouse tail: Lymphatic hyperplasia, VEGF-C upregulation, and the protective role of MMP-9. Microvascular Research. 2006 Jul.

96. Breslin JW, Gaudreault N, Watson KD, Reynoso R, Yuan SY, Wu MH. Vascular endothelial growth factor-C stimulates the lymphatic pump by a VEGF receptor-3-dependent mechanism. American Journal of Physiology: Heart and Circulatory Physiology. 2007 Jul; 293(1):H709-18. [PubMed: 17400713] 


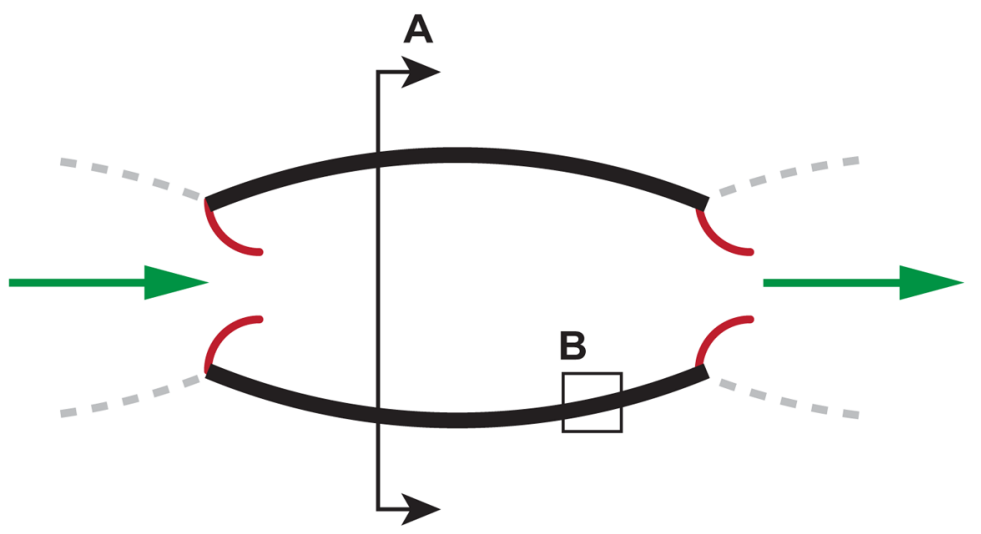

A

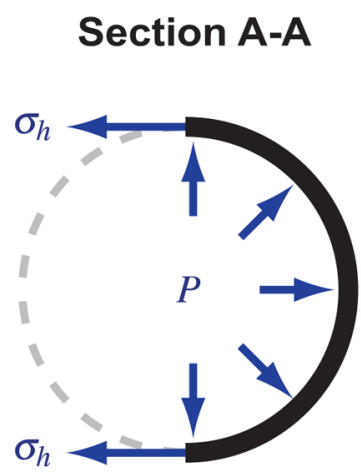

Region B

- Lymph flow direction

- Hoop stress and transmural pressure

- Axial stress and fluid shear stress

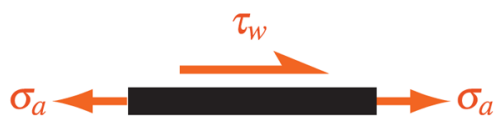

Fig. 1.

Collecting lymphatics consist of individual contractile units separated by valves, termed lymphangions. The contractile activity produces several time-varying loads on the vessel, many of which are thought to play a role in regulating lymphatic contraction. 